\title{
Research for Improving Patient Care
}

\author{
POOJA DEWAN ${ }^{1}$ AND HPS SACHDEV ${ }^{2}$ \\ From Department of Pediatrics, ${ }^{1}$ University College of Medical Sciences, Delhi; and ${ }^{2}$ Department of Pediatrics and Clinical \\ Epidemiology, Sitaram Bhartia Institute of Science and Research, New Delhi; India. \\ Correspondence to: Dr. Pooja Dewan, Parsvnath Prestige 2, Sector 93A, Noida, Uttar Pradesh, India. poojadewan@hotmail.com
}

Research is an integral part of medicine. Health research aims at generating evidence for development of new medicines, procedures and tools, all of which are directed at improved patient care. Research also aims at incorporating this evidence into clinical practice by developing clinical practice guidelines and developing robust health systems including governmental policies and systems. Health research not only allows doctors to decide how to best treat patients but also empowers patients to take active role in their health.

Keywords: Evidence-based medicine, Health system, Levels of Evidence, Translational medicine.

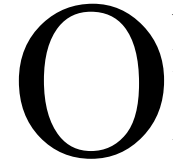
ver centuries, health research has relied heavily on keen observation, clinical experience and meticulous planning, all of which has led to evolution of healthcare and innovation in patient care, as is illustrated well by two historical vignettes.

The eighteenth century saw a serendipitous breakthrough in medicine when Edward Jenner, an English physician, discovered the smallpox vaccine based on his observation that milkmaids who developed cowpox were protected from smallpox in due course [1]. Although this was a simple observation, it reiterates the fact that medical science is built out of an eye for detail and a thinking mind.

The importance of structured research including rational use of numbers in medical science can be exemplified by Lind's 'Salisbury experiment' in 1747, largely cited as the first clinical trial [2]. Although, Lind must be commended for using comparable study groups (six therapeutic groups of two participants each) under controlled environment, his observation that oranges and lemons cured scurvy in only one group seems more like a fortuitous discovery. Lind did establish citrus fruits as anti-scorbutic, but his success may simply have been because only one out of the six cures he used had vitamin $\mathrm{C}$ whereas the other five did not. Decades later, when lemon juice was unintentionally swapped by lime-juice among sailors on polar explorations, scurvy resurfaced questioning the validity of Lind's trial; for limes contain only half as much vitamin $\mathrm{C}$ as lemons and oranges, a fact unknown then.

These examples highlighted that traditional health research, which was largely instinctive, experiencebased, riddled with elements of bias, unforeseen errors and confounding variables; needs to be supplanted with robust evidence-based research. Experience-based (nonformal) research can also be misleading and exploitative in the garb of science. The enticing advertisements which manipulate the population beliefs of diet-based or natural remedy-based wellness like supplements to enhance energy and height are clearly logically fallacious and morally repugnant.

While the above examples can be used to scoff at traditional beliefs, the real aim of health research goes much beyond. Health research makes possible the development of new medicines, new procedures and new tools possible which empower the doctors to treat their patients better. Research intends to improve patient outcomes by minimizing error and ensuring a standard of care [3]. Herein, we intend to sensitize the readers to the importance of formal and planned investigations for improving patient care, and how to interpret research.

\section{Types of Health Research}

Health research can be categorized as primary or secondary research. Primary research involves conducting studies and collection of raw data by the research team. Secondary research like systematic reviews and meta-analysis involve analysis and synthesis of research conducted by others and could yields results with less cost and sometimes in relatively shorter span of time for the consumption of the readers, although these involve tedious and intense efforts on part of the researcher. Primary health research can be further categorized as basic (bench or laboratory) 
research, clinical research and epidemiological research. Basic research includes experimentation in pre-clinical fields such as molecular biology, medical genetics, immunology, animal studies and laboratory research, which provide an understanding into the anatomy, physiology and cellular biology of health and disease, or the biological basis of disease (for example, the effect of copper on oxidative stress in chicken hepatocytes). Clinical research includes interventional studies (clinical trials) or observational studies (cross-sectional, case control, cohort, retrospective, case series), which evaluate disease characteristics, drugs, devices, and diagnostic tools intended for therapeutic, preventive or diagnostic purpose for a disease condition. Examples include: Therapeutic efficacy of intramuscular versus oral vitamin B12 in megaloblastic anemia, association of celiac disease and rickets, and hematological effect of iron supplementation in breast fed term low birth weight infants. Epidemiological studies are population-based (interventional or observational) and aim to identify the cause and distribution of diseases [4]; for example, etiology of pyrexia of unknown origin amongst children from a tribal belt of central India. Health research may also be categorized as qualitative or quantitative [5]. Quantitative research involves direct measurements. Qualitative research is concerned with understanding human behavior from the informant's perspective wherein data are collected by interviews or observation and analyzed by themes derived from participant's description. Research methods can also be categorized as descriptive or analytical.

\section{Choosing a Research Topic: POE Vs DOE}

Research should seek to address queries encountered in day-to-day practice. Concerns like morbidity, mortality, or quality of life (for example, oral vitamin D supplementation for reducing severity of pneumonia in under-five children) which matter to both treating physicians and patients are referred to as PatientOriented Evidence (POE) or Patient-Oriented Evidence that Matters (POEMs) [6]. Evidence related to incidence, prevalence, etiology, pharmacology, or pathophysiology of disease generate "Disease-Oriented Evidence" (DOE) which are often of greater interest to researchers or laboratory medicine specialists (for example, telomere shortening in leukocytes of children with Human Immunodeficiency Virus).

Research should also focus on the needs of the local population (regionally responsive) and provide solutions which are acceptable, efficacious, safe and sustainable for the region. Most of the low- and middle-income countries (LMICs) could not reap the expected benefits from research conducted or innovations developed in the western countries as the products so developed were either too costly or were not suited to their needs. For example, access to immunotherapy for children with leukemia remains out of reach in most developing countries due to the costs, regulatory barriers, and limited availability of trained health care personnel.

Several pioneering works by Indian physicians can serve as role models for integrating research into office practice and offer need-based solutions in the Indian and other LMIC contexts. Starting from the mid-twentieth century, Dr. Chatterjee can be credited for publishing the first paper on use of oral rehydrating therapy for cholera in humans [7]. Subsequently, contributory efforts of Indian clinicians [8-10] established the therapeutic role of oral zinc in acute diarrhea in under-five children, which has since been adopted by WHO as a standard of care alongside oral rehydration solution. Establishing room air as the modality of choice for resuscitating asphyxiated neonates has been another pioneering work [11]. The development of Rotavac $\AA$, a low-cost indigenous rotavirus vaccine is also a noteworthy innovation [12]. Some other researchers helped shape the guidelines for management of regional public health problems like rickettsial illness [13], and scorpion envenomation [14], and unfold the mystery behind hypoglycemic encephalopathy which was perplexing for the physicians in Western Uttar Pradesh [15]. All these indigenous research works and many more are embodiment of the quote "With every lock comes a key".

\section{Planning Research: Methods and Designs}

Depending upon the research question, a particular research method may be more suited. For example, questions about people's perceptions of COVID-19 illness may be answered by qualitative research, but the efficacy of a drug like Remdesvir in the treatment of severe COVID-19 illness are better enunciated through a clinical trial or a case-control design. All these research methods are generally used to complement each other in order to understand a phenomenon as completely, as feasible. Fig. 1 summarizes the various types of research designs that can be employed.

\section{Evidence-based Medicine (EBM)}

Clinical decisions backed by scientific evidence inspire confidence and conviction to the clinician. Levels of evidence (LOE) are assigned to studies based on the methodological rigor of their design, validity, and relevance to patient care. These levels are arranged in a hierarchical fashion to depict an Evidence Pyramid [16]. The studies like the systematic reviews and meta-analysis 


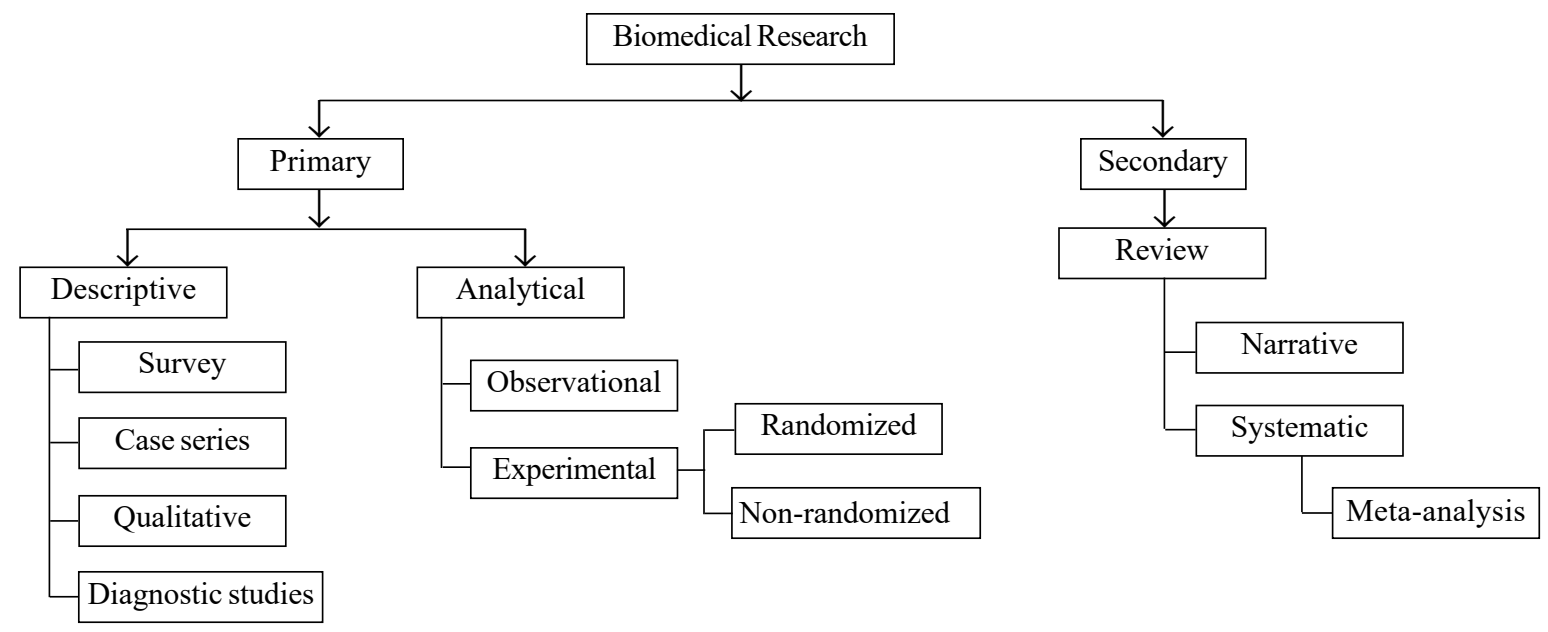

Fig. 1 Study designs and methods used in biomedical research.

or homogenous randomized controlled trials (RCTs), are regarded as the highest quality and are placed at the apex of the pyramid (Level 1 evidence). Observational research is lower and expert opinion is at the lowest rung (Level 5 evidence) as depicted in Fig. 2. The type of available evidence is dependent upon the research question and may not always be the highest level of evidence. Some studies would be deemed unethical for RCT design; for example, randomizing newborn infants to receive breastfeeding or a formula feed for determining effect on body composition. In such situations, it is best to work the way down the Evidence Pyramid to the next highest level of evidence.

\section{Scrutiny of Evidence}

RCTs are the often allocated the highest level of evidence, however, their respective results should be carefully scrutinized with respect to their power, bias or other types of errors. For example, an underpowered RCT on vitamin D supplementation for decreasing severity of pneumonia in under-five children may yield a negative result when in fact vitamin D supplementation could be beneficial. The assessment criteria as outlined in the Cochrane Handbook for Systematic Reviews of Interventions [17], may be used to help ascertain the methodological rigor of a RCT; factors like sequence generation (selection bias), absence of allocation sequence concealment (selection

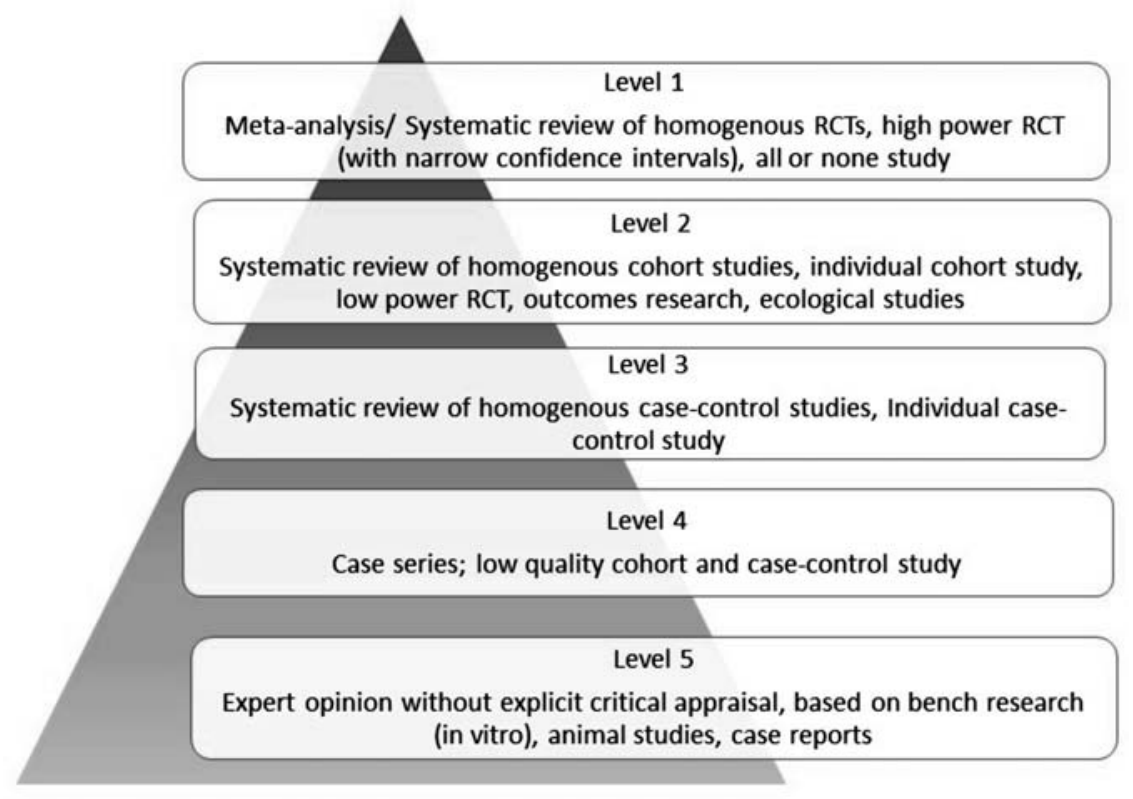

Fig. 2 Hierarchy of study designs and levels of clinical evidence. 
bias), lack of blinding of participants and personnel (performance bias), not blinding the assessors of outcome (detection bias), missing outcome data (attrition bias) and selective reporting or deliberate suppression of outcome (reporting bias) need consideration. Presence of these biases undermines the confidence in interpretation and conclusions. Likewise, for observational studies, confounding variables and bias need to be ascertained using the detailed Risk of Bias in Non-randomized Studies of Interventions (ROBINS-I) tool [18].

\section{Grading of Evidence}

It is important for a clinician to not only keep abreast of the latest research but also be aware of limitations of the studies. Hence, a grading system (grades A, B, C and D) is in place to assist the clinician for decision-making and adopting evidence into practice [19]. This grading takes into account the research question, the available scientific evidence including their methodological rigors and limitations like imprecision, inconsistency, indirectness of study results, and publication bias. It does not downgrade lower level evidence when deciding recommendations provided the results are consistent. For example, a strong recommendation (grade A) is given to both level I evidence as well as consistent evidence from Levels II, III and IV studies. Consistent level 2 or 3 studies and extrapolation from level 1 studies are given grade B. Level 4 studies and extrapolation from level 2 or 3 studies are allotted grade C. Level 5 evidence and inconsistent or inconclusive studies from any level are the lowest grade (grade D).

\section{Bringing Published Research into Practice}

It is important to critically evaluate published research before adopting it into practice. While it is good to consider the methodological rigor of published data, yet what gets published is not always sacrosanct. Journals often publish only a small fraction of the studies while most of the studies with negative results (i.e., those failing to yield statistical significance) remain in closets and drawers. Publication bias, predatory editorial practices, and ritualistic statistical practices can all be counterproductive to EBM. Clinical practice decisions should not be based on a single study. Clinical decisions based on low-quality evidence can unintentionally lead to alarming consequences for the patient. Considerations of cost, regional applicability (similarity between your health set up and the study setting), and benefits of change compared to the risks, and legal implications should be considered in addition to the merits of study design and its results. Although "to err is human", it is unacceptable that health care system should end up harming the patients whom it is designed to protect.

\section{Responsible Conduct of Research in Minors}

Children are a vulnerable group with their own specific needs and hence research needs to be focused to address their unique issues. Research in children is also challenging as these young participants need to be specially protected while we seek answers to clinical questions and issues which can offer them benefit. Growth, development and childhood illnesses are research areas which are well recognized in this group. Alongside, focused research on neonatal health and disease and adolescent health are also addressed by including these specific cohorts as research participants. However, children should be research participants only if the research question can be answered by their participation. Research should always be conducted in adults before children unless the disease is unique to pediatric age group like Kawasaki disease, birth asphyxia, neuroblastoma, congenital adrenal hyperplasia, etc. Likewise, issues pertaining to neonates like interventions for neonatal resuscitation can only be addressed by studying neonates in research. It is important to remember that findings of research on adults should not be extrapolated to children. For example, dose of a particular drug (established in adults) in a child weighting one-half of the adult weight cannot be simply axed to half! However, despite the need for pediatric research, it has been a low priority at policy level. Ethical constraints (limited ability of the child to understand the implications of research, coercion, guardianship and autonomy of the child), lack of funding, and reluctance by community are some major impediments to research in children. Consequently, recommendations in children are often derived from adult studies or no studies at all!

\section{Translational Medicine: Bench to Bedside}

Evidence-informed practice is critical to delivery of optimum healthcare. Any lag in the translation of research into practice can lead to overuse, underuse, and misuse of evidence [20]. Overuse refers to the continued use of ineffective interventions or interventions with a scope for greater harm than advantage; for example, empirical use of antibiotics in all preterm neonates admitted to the intensive care unit. Failure to provide treatment despite robust scientific evidence would constitute underuse; for example, failure to prescribe zinc supplements for acute dehydrating diarrhea in toddlers. Misuse would occur when the prescribed treatment is neither apt nor safe leading to adverse health effects; for example, routine prescription of oral steroids in all wheezy children. Translational medicine aims at bridging this 'Know-Do gap' by incorporating evidence-based findings into office practice in a timely manner. Translational researchers use 


\section{KEY MESSAGES}

- Research should be relevant, participatory, and patient-oriented.

- A critical appraisal of the research methodology and its validation is imperative before translating the findings into routine clinical practice.

- Evidence-based medicine, translational medicine and health systems research are the triology of biomedical research.

secondary research methods to scale up the application of scientific evidence to the level of the healthcare system rather than the individual healthcare providers.

\section{Implementation Science: Challenges and the Road Ahead!}

Implementation science is the "scientific study of methods to promote the systematic uptake of evidencebased research into routine practice, and, hence, to improve the quality and effectiveness of health services and care" [21]. Development of clinical practice guidelines (CPGs) and their dissemination is one of the most tactical methods to translate evidence into practice. However, it is important to consider that mere publication of CPGs cannot ensure change in clinical practice. Simultaneous publication of overlapping guidelines by different scientific bodies, rapidly emerging scientific evidence rendering the existing guidelines redundant (for example, therapeutic use of hydroxychloroquine in COVID-19), trust in the validity of current practice methods, contradictory information from different information sources, lack of awareness among practicing physicians, personal bias of the physicians based on experience, and poor health systems constitute major barriers to implementation of CPGs into practice. Numerous obstacles can impede conduct of clinical trials in LMICs like the lack of infrastructure, heterogeneity of resource availability among countries, unfamiliarity with clinical trial regulations, cultural/ethical issues, and other legal constraints around data-sharing. Only about $10 \%$ of the global expenditure on health research and development was used for research in the developing countries where $90 \%$ of all preventable global deaths had occurred [22]. A mere $0.09 \%$ of the gross domestic product (GDP) in India was spent on health research in 2011-12 and only a meager $0.02 \%$ was available from public sources [23].

Despite evidence to show that patients treated at research-active hospitals fare better [24,25], many hospitals have not been able to completely integrate research into clinical care across all their specialties. Mistrust in health systems and fear of being exploited prevents patients from participating in research. Lack of financial incentives, miniscule funding for research, no protected time for research, high expectations of patients, societal norms, complacency of physicians developed through years of traditional practice, fear of litigation hassles, cumbersome patent laws, and ethical and regulatory clearances, deter most practicing doctors from participation in research.

To foster improvement in health research, it is imperative to understand the magnitude of the 'Know-do gap' at the level of providers and institutions, and augment the dissemination of evidence from health research, infrastructure, funding and priority-setting for health research. The setting up of Department of Health Research (DHR) by the Ministry of Health and Family Welfare in 2007 has been a welcome step to this effect. Innovation in the medical education system coupled with greater use of digital technology in research as well as healthcare delivery are needed. E-medicine or telemedicine can aid healthcare delivery. A change in the attitudes of healthcare providers and patients is also needed wherein patients are treated as clients. Patients need to participate not only in their own healthcare decisions but also in research through informed consent. A more participatory role of the patients and an overall positive outlook of the clinicians to be involved in research will ensure a more robust health system in the future.

Funding: None; Competing interests: None stated.

\section{REFERENCES}

1. Smith KA. Edward Jenner and the small pox vaccine. Front Immunol. 2011;2:21.

2. Hughes RE. James Lind and the cure of scurvy: An experimental approach. Med Hist. 1975;19:342-51.

3. Krzyzanowska MK, Kaplan R, Sullivan R. How may clinical research improve healthcare outcomes? Ann Oncol. 2011;22 Supp1 7:vii10-vii15.

4. Röhrig B, du Prel JB, Wachtlin D, et al. Types of study in medical research: Part 3 of a series on evaluation of scientific publications. Dtsch Arztebl Int. 2009;106:262-8.

5. Lakshman M, Sinha L, Biswas M, et al. Quantitative vs qualitative research methods. Indian J Pediatr. 2000; $67: 369-77$ 
6. Smith R. A POEM a week for the BMJ. BMJ. 2002; 325:983.

7. Chatterjee HN. Therapy of diarrhoea in cholera. Lancet. 1953;265:1045-6.

8. Sachdev HP, Mittal NK, Mittal SK, et al. A controlled trial on utility of oral zinc supplementation in acute dehydrating diarrhea in infants. J Pediatr Gastroenterol Nutr. 1988;7: 877-81.

9. Bhatnagar S, Bahl R, Sharma PK, et al. Zinc with oral rehydration therapy reduces stool output and duration of diarrhea in hospitalized children: A randomized controlled trial. J Pediatr Gastroenterol Nutr. 2004;38:34-40.

10. Bahl R, Bhandari N, Saksena M, et al. Efficacy of zincfortified oral rehydration solution in 6 to 35 month old children with acute diarrhea. J Pediatr. 2002;141:677-82.

11. Ramji S, Rasaily R, Mishra P, et al. Resuscitation of asphyxiated newborns with room air or $100 \%$ oxygen at birth: A multicentric clinical trial. Indian Pediatr. 2003; 40:510-17.

12. Ella R, Bobba R, Muralidhar S, et al. A Phase 4, multicentre, randomized, single-blind clinical trial to evaluate the immunogenicity of the live, attenuated, oral rotavirus vaccine (116E), ROTAVAC $\AA$, administered simultaneously with or without the buffering agent in healthy infants in India. Hum Vaccin Immunother. 2018;14:1791-1799.

13. Rathi NB, Rathi AN, Goodman $\mathrm{MH}$, et al. Rickettsial diseases in central India: Proposed clinical scoring system for early detection of spotted fever. Indian Pediatr. 2011;48:867-72.

14. Vashishtha VM, Kumar A, John TJ, et al. Cassia occidental is poisoning causes fatal coma in children in western Uttar Pradesh. Indian Pediatr. 2007;44:522-5.

15. Kale AA. A crusade against scorpion sting: Life and works of Dr. Himmatrao Bawaskar. J Family Med Prim Care. 2012;1:52-5.

16. Oxford Centre for Evidence-Based Medicine: Levels of Evidence (March 2009). Accessed on October 31, 2020.
Available from: https://www.cebm.ox.ac.uk/resources/ levels-of-evidenceloxford-centre-for-evidence-basedmedicine-levels-of-evidence-march-2009.

17. Higgins JPT, Thomas J, Chandler J, et al (editors). Cochrane Handbook for Systematic Reviews of Interventions version 6.1 (updated September 2020). Cochrane, 2020. Accessed November 1, 2020. Available from: http://www.training. cochrane.org/handbook.

18. Sterne JAC, Higgins JPT, Elbers RG, et al and the development group for ROBINS-I. Risk Of Bias In Nonrandomized Studies of Interventions (ROBINS-I): Detailed guidance, updated 12 October 2016. Available from: $h t t p: / /$ www.riskofbias.info. Accessed October 26, 2020.

19. Balshem H, Helfand M, Schünemann HJ, et al. GRADE guidelines: 3 rating the quality of evidence. J Clin Epidemiol. 2011;64:401-6.

20. Institute of Medicine. Crossing the quality chasm: A new health system for the 21 st century. 2001. Accessed October 30, 2020. Available at: http://www.nationalacademies.org/ hmd/w/media/Files/Report\%20Files/2001/Crossing-theQuality-Chasm/Quality\%20Chasm\%202001\%20\%20 report\%20brief. pdf

21. Eccles MP, Mittmann BS. Welcome to implementation science. Implem Sci. 2006;1:1.

22. Kilama WL. The 10/90 gap in sub-Saharan Africa: Resolving inequities in health research. Acta Trop. 2009;112:S8-S15.

23. Dandona L, Dandona R, Kumar GA, et al. Mapping of health research funding in India. Natl Med $\mathrm{J}$ India. 2017;30:309-16.

24. Downing A, Morris EJ, Corrigan N, et al. High hospital research participation and improved colorectal cancer survival outcomes: A population-based study. Gut. 2017;66:89-96.

25. Nijjar SK, D'Amico MI, Wimalaweera NA, et al. Participation in clinical trials improves outcomes in women's health: A systematic review and meta-analysis. BJOG. 2017;124:863-71. 